\title{
Inmigración y educación intercultural en la formación inicial docente*
}

\author{
Immigration and intercultural education in the initial teacher training \\ Imigração e educação intercultural na formação inicial docente
}

\author{
Alejandra Sánchez B., ${ }^{\mathrm{a}}$ Leandro Navas M., ${ }^{\mathrm{b}}$ Pablo Holgado T. ${ }^{\mathrm{c}}$ \\ ${ }^{a}$ Escuela de Pedagogía en Educación Diferencial, Universidad Austral de Chile. Correo electrónico: asan- \\ chezb@spm.uach.cl. Telf.: 065. 277173 \\ bDepartamento de Psicología Evolutiva y Didáctic, Universidad de Alicante. Correo electrónico: \\ leandro.navas@ua.es. Telf.: 34965903400 (ext. 2913)
}

cFacultad de Psicología, Universidad Nacional de Educación a Distancia. Correo electrónico: pfholgado@psi. uned.es. Telf.: 34913988648

\begin{abstract}
RESUMEN
El objetivo del estudio fue analizar las actitudes hacia la inmigración de un grupo de estudiantes de pedagogía chilenos. Participaron 720 sujetos. Los resultados indicaron que las actitudes muestran una tendencia favorable, excepto para mantener una relación permanente o íntima. Un número importante de sujetos mostró una actitud intermedia, la cual se podría relacionar con el prejuicio sutil. Existen diferencias en las actitudes asociadas a la experiencia de contacto con inmigrantes.
\end{abstract}

Palabras clave: actitud, inmigración, inmigrante, prejuicio.

\begin{abstract}
The aim of this study was to analyse the attitude toward immigration of a group Chilean students in teacher training. 720 subjects participated. Results indicated positive trend, excepting when maintaining a permanent or intimate relationship. A significant number of subjects showed an intermediate attitude, which could be related to subtle prejudice. There are differences in attitude associated with the experience of contact with immigrants.
\end{abstract}

Key words: attitude, immigration, immigrant, prejudice.

\section{RESUMO}

Objetiva-se analisar atitudes em relação à imigração de um grupo de estudantes chilenos da Pedagogia. Participaram 720 sujeitos. Resultados indicaram que as atitudes mostram uma tendência favorável, exceto para manter um relacionamento permanente ou íntimo. Um número significativo de sujeitos mostrou uma atitude intermediária que pode relacionar-se ao preconceito sutil. Existem diferenças nas atitudes associadas à experiência de contato com imigrantes.

Palavras chave: atitude, imigração, imigrantes, preconceito. 


\section{INTRODUCCIÓN}

Quizás, uno de los desafíos más importantes del siglo XXI sea conseguir una convivencia armónica entre los individuos, en una sociedad que es cada vez más heterogénea culturalmente y, por lo tanto, potencialmente más conflictiva. Sin embargo, dicha aspiración no es algo fácil de concretar, ya que es habitual que en el centro de las relaciones interculturales se observen una serie de estereotipos y prejuicios que tiñen las interacciones grupales con hostilidad y discriminación. En Chile, el último censo de población señala que el flujo migratorio aumentó en un 75\% durante el periodo comprendido entre 1992 y 2002 (Chile, Instituto Nacional de Estadísticas, 2003). Hoy, alrededor del 2\% de la población chilena está constituida por extranjeros y la actual Política Nacional Migratoria reconoce a Chile como un país receptor de cuotas significativas de inmigrantes (Chile, Presidencia de la República, 2008). El censo de 2002, además, arrojó los primeros datos de una realidad emergente, un total de 34.006 niños extranjeros menores de 14 años residen en Chile; de estos, el 73,9\% corresponde a niños procedentes de diferentes países de América del Sur.

La inmigración y la interculturalidad en la escuela son fenómenos recientes en la sociedad chilena que no están suficientemente considerados en la legislación actual. Tanto es así que solo a partir del año 2005 se reguló el ingreso de los niños inmigrantes al sistema educativo. Sin embargo, según Quesille (2007), el acceso a los establecimientos educacionales se ve dificultado, principalmente, por el desconocimiento que los administradores tienen de la legislación vigente y porque lo que parece ser un trámite sencillo de realizar, finalmente, resulta engorroso, por el gran número de documentos que se deben presentar para conseguir una matrícula.

Los países que se inician en temas de inmigración, como es el caso de Chile, deben tener presente que los resultados que, de manera consistente, se repiten generalmente en las escuelas que atienden alumnos procedentes de otras culturas, son: que estos presentan un rendimiento académico por debajo del promedio, dificultades en el desarrollo de la identidad étnica y problemas de inclusión escolar (Aboud, 1988; Glick y Hohmann-Marriott, 2007; Glick y White, 2003; Porter y MacLeod, 1996). En las décadas de 1960 y 1970, algunos teóricos explicaban los problemas escolares recurriendo al modelo del déficit cultural, cuyo supuesto era que la cultura familiar de los estudiantes era inferior porque no los preparaba para adaptarse a la escuela. En la actualidad, la mayoría de las personas rechaza la idea de los déficit culturales, ya que se considera que no existen culturas deficientes, sino que se trata de incompatibilidades entre la cultura familiar del estudiante y las expectativas de la escuela (Gallimore y Goldenberg, 2001). Las explicaciones más recientes de por qué los estudiantes de minorías culturales presentan bajo rendimiento académico, otorgan un lugar importante a las bajas expectativas, a los prejuicios y a los estereotipos de los maestros y de los demás estudiantes, así como también a las políticas escolares no inclusivas de los centros educativos (Woolfolk, 2006).

Para Díaz, Baraja y Royo (1996), el estilo de atención a la diversidad de la inmensa mayoría de los profesores se caracteriza por ser pasivo, es decir, dejan en mano de los alumnos el control de las interacciones. Esto indica que no intentan compensar la desigualdad de oportunidades que, en este sentido, imponen las propias diferencias culturales de los alumnos, mas tampoco las fomentan. Díaz (2002) señala que esta extendida forma de tratar la diversidad cultural, actuando como si no existiera, suele observarse en los profesores cuando manifiestan que todos los alumnos son iguales y que por eso no es 
necesario hacer diferencias entre ellos. Para Jordan (1994) esta forma de atender las diferencias culturales en el aula, por una parte, expresa el deseo de los maestros de no ser considerados discriminadores, lo que ha denominado la ilusión del trato igualitario, pero también deja ver la falta de recursos de los docentes para adaptarse a la multiculturalidad de los estudiantes. Quizás la forma más adecuada de cambiar las actitudes discriminatorias de los profesores hacia los estudiantes de otras culturas sea modificar sus actitudes durante el proceso de formación inicial.

Contradictoriamente, el sistema de educación superior chileno parece permanecer ajeno a estas demandas de formación o, al menos, eso parece desprenderse del hecho que no se considere explícitamente necesario incluir en los programas de formación de profesores, una educación sistemática que permita a los futuros maestros capacitarse para atender las conductas diferentes, derivadas de adecuaciones culturales diversas, y aproximarse con menos prejuicios al alumnado con condicionantes culturales diferentes.

Teniendo en cuenta estos antecedentes, parece oportuno intentar identificar las ideas, percepciones y actitudes que manifiestan un grupo de maestros en formación hacia la inmigración y los inmigrantes.

\section{MÉTODO}

\subsection{PARTICIPANTES}

Participaron 720 profesores en formación pertenecientes a tres universidades localizadas en la octava y novena región del país. La técnica de selección fue muestreo no probabilístico. Participaron 488 mujeres y 232 hombres y el rango de edad oscila entre 18 y 33 años $(M=21,13$; DT = 2,35). Los sujetos se distribuían en nueve especialidades y en cursos que van desde $1^{\circ}$ a $5^{\circ}$ año. El 73,7\% señaló tener algún tipo de relación con personas inmigrantes como compartir barrio, compartir trabajo o estudios, participar en actividades sociales o mantener una relación de amistad o noviazgo.

\subsection{INSTRUMENTOS Y VARIABLES}

El instrumento empleado para describir las actitudes de los futuros docentes es la Escala de Actitudes Hacia la Inmigración (EAHI) de León, Mira y Gómez (2007). El instrumento fue adaptado al contexto chileno, los análisis de la estructura factorial y las propiedades psicométricas de la escala adaptada son aceptables (Holgado, Sánchez y Navas, 2011).

El instrumento incluye 30 elementos que los sujetos han de evaluar en una escala de 5 puntos en la que 0 indica "nada de acuerdo" y 4 significa "muy de acuerdo". Permite evaluar las siguientes variables:

- Derechos Básicos (DB) que se refiere a las políticas conducentes a garantizar los derechos fundamentales de las personas inmigrantes. Explica el 8,81\% de la varianza de la escala y presenta un coeficiente de fiabilidad de 0,85 .

- Actitud de rechazo (AR) que representa la actitud de evitación o rechazo hacia personas inmigrantes, explica el $8,55 \%$ de la varianza y presenta un coeficiente de fiabilidad de 0,77 . 
- Políticas de Igualdad (PI), explica el 8,01\% de la varianza de la escala y presenta un índice de fiabilidad de 0,77 y se refiere al reconocimiento de la igualdad de oportunidades entre chilenos e inmigrantes.

- Rasgos Negativos (RN), explica el 7,75\% de la varianza y su consistencia interna es 0,73 . Se refiere a aspectos negativos asociados a las personas inmigrantes.

- Actitud de tolerancia (AT) que hace referencia a una actitud de aceptación de interacción y convivencia con personas inmigrantes. Explica el 6,90\% de la varianza y el coeficiente de fiabilidad es 0,73 .

- Actitud de aproximación (AA) que explica el 5,88\% de la varianza de la escala, el coeficiente alfa de Cronbach es 0,79 y hace referencia a la intención de mantener relaciones íntimas con personas inmigrantes.

- Rasgos positivos (RP) que representa aspectos positivos asociados a las personas inmigrantes. Explica el 5,61\% de la varianza y la consistencia interna es 0,60.

- Exclusión (EX) que se refiere a dificultades a las que se enfrentan los inmigrantes en Chile, explica el 5,23\% de la varianza de la escala y el coeficiente de fiabilidad es 0,74.

\subsection{PROCEDIMIENTO}

La aplicación del instrumento fue realizada por un encuestador, previamente instruido. Siguiendo los criterios de la Comisión Nacional de Investigación Científica y Tecnológica de Chile (CONICYT), a los participantes se les ofreció información sobre los objetivos de la investigación y sobre los investigadores responsables, así como también una dirección de correo electrónico para realizar consultas relativas al estudio; también se les solicitó su colaboración destacando que la participación era voluntaria y anónima. Tras conseguir el consentimiento verbal, en una sesión y en su aula habitual, los participantes respondieron al cuestionario, contaron con la asistencia del encuestador para aclarar sus dudas y no recibieron retribución. Los resultados del estudio fueron remitidos a las universidades de procedencia. Con el fin de evitar confusiones conceptuales entre minorías étnica autóctonas (mapuches, aymaras, etc.) y minorías étnicas extranjeras (peruanos, bolivianos, colombianos, argentinos, etc.), se instruyó en forma verbal y escrita a los participantes.

\subsection{ANÁLISIS DE LOS DATOS}

Se llevaron a cabo análisis descriptivos, de frecuencias y de contrates de medias utilizando la prueba t de Student. Para ello se utilizó el paquete estadístico SPSS (15.0).

\section{RESULTADOS}

En la Tabla 1 se ofrecen los estadísticos descriptivos de las variables antes mencionadas. Los resultados muestran que los valores de asimetría y curtosis están comprendidos entre $-1,96$ y $+1,96$, lo cual indica que las variables analizadas se ajustan razonablemente a la distribución normal.

En la Tabla 2 se presenta un resumen del análisis de frecuencias de respuestas por variables, estos resultados nos aportan información sobre las actitudes que manifiestan los participantes hacia la inmigración. Hay que considerar que mientras más bajo sea el puntaje, más positiva es la actitud. 
Tabla 1. Análisis descriptivos de variables de actitudes hacia la inmigración

\begin{tabular}{|c|c|c|c|c|c|}
\hline Variable & $\boldsymbol{M}$ & $\boldsymbol{D E}$ & $\boldsymbol{V}$ & $\boldsymbol{A}$ & $\boldsymbol{C}$ \\
\hline Derechos básicos & 4,01 & 3,51 & 12,31 & 0,88 & 0,50 \\
\hline Políticas de igualdad & 10,40 & 5,34 & 28,48 & 0,16 & $-0,46$ \\
\hline Rasgos positivos & 5,1 & 2,04 & 4,16 & 0,02 & 0,02 \\
\hline Rasgos negativos & 5,82 & 3,36 & 11,29 & 0,01 & $-0,42$ \\
\hline Actitud de tolerancia & 7,84 & 3,61 & 13,06 & $-0,64$ & $-0,50$ \\
\hline Actitud de aproximación & 4,48 & 1,37 & 1,89 & $-0,04$ & 0,58 \\
\hline Actitud de rechazo & 2,89 & 3,59 & 12,85 & 1,15 & 0,61 \\
\hline Exclusión & 3,62 & 2,76 & 7,64 & 0,17 & $-1,23$ \\
\hline
\end{tabular}

$\mathrm{M}=$ Media; $\mathrm{DE}=$ Desviación estándar; $\mathrm{V}=$ Varianza; $\mathrm{A}=$ Asimetría $; \mathrm{C}=$ Curtosis .

Tabla 2. Análisis de frecuencias por variables

\begin{tabular}{|c|c|c|c|c|c|c|}
\hline \multirow[t]{2}{*}{ Variable } & \multicolumn{2}{|c|}{ Actitud positiva } & \multicolumn{2}{|c|}{ Actitud intermedia } & \multicolumn{2}{|c|}{ Actitud negativa } \\
\hline & $f$ & $\%$ & $f$ & $\%$ & $f$ & $\%$ \\
\hline \multirow{2}{*}{$\begin{array}{l}\text { Derechos básicos } \\
\quad(\mathrm{R}=0-16)\end{array}$} & 438 & 60,8 & 254 & 35,3 & 28 & 3,9 \\
\hline & \multicolumn{2}{|c|}{$(\mathrm{R}=0-4)$} & \multicolumn{2}{|c|}{$(\mathrm{R}=5-11)$} & \multicolumn{2}{|c|}{$(\mathrm{R}=12-16)$} \\
\hline \multirow{2}{*}{$\begin{array}{c}\text { Políticas de } \\
\text { igualdad }(\mathrm{R}=0 \text { - } \\
\text { 24) }\end{array}$} & 216 & 30,0 & 406 & 56,3 & 98 & 13,7 \\
\hline & \multicolumn{2}{|c|}{$(\mathrm{R}=0-7)$} & \multicolumn{2}{|c|}{$(\mathrm{R}=8-16)$} & \multicolumn{2}{|c|}{$(\mathrm{R}=17-24)$} \\
\hline \multirow{2}{*}{$\begin{array}{l}\text { Rasgos positivos } \\
\qquad(\mathrm{R}=0-12)\end{array}$} & 175 & 24,3 & 498 & 70,5 & 37 & 5,2 \\
\hline & \multicolumn{2}{|c|}{$(\mathrm{R}=0-3)$} & \multicolumn{2}{|c|}{$(\mathrm{R}=4-8)$} & \multicolumn{2}{|c|}{$(\mathrm{R}=9-12)$} \\
\hline \multirow{2}{*}{$\begin{array}{l}\text { Rasgos negativos (R } \\
\quad=0-20)\end{array}$} & 402 & 55,8 & 313 & 43,3 & 5 & 0,9 \\
\hline & \multicolumn{2}{|c|}{$(\mathrm{R}=0-6)$} & \multicolumn{2}{|c|}{$(\mathrm{R}=7-13)$} & \multicolumn{2}{|c|}{$(\mathrm{R}=14-20)$} \\
\hline \multirow{2}{*}{$\begin{array}{c}\text { Actitud de } \\
\text { tolerancia }(\mathrm{R}=0 \\
-12) \\
\end{array}$} & 96 & 13,3 & 304 & 42,2 & 320 & 44,5 \\
\hline & \multicolumn{2}{|c|}{$(\mathrm{R}=0-3)$} & \multicolumn{2}{|c|}{$(\mathrm{R}=4-8)$} & \multicolumn{2}{|c|}{$(\mathrm{R}=9-12)$} \\
\hline \multirow{2}{*}{$\begin{array}{c}\text { Actitud de } \\
\text { aproximación }(\mathrm{R}= \\
0-8)\end{array}$} & 96 & 13,3 & 304 & 42,2 & 320 & 44,5 \\
\hline & \multicolumn{2}{|c|}{$(\mathrm{R}=0-3)$} & \multicolumn{2}{|c|}{$(\mathrm{R}=4-8)$} & \multicolumn{2}{|c|}{$(\mathrm{R}=9-12)$} \\
\hline \multirow{2}{*}{$\begin{array}{l}\text { Actitud de rechazo } \\
\quad(\mathrm{R}=0-20)\end{array}$} & 588 & 81,7 & 129 & 17,8 & 3 & 0,5 \\
\hline & \multicolumn{2}{|c|}{$(\mathrm{R}=0-6)$} & \multicolumn{2}{|c|}{$(\mathrm{R}=7-13)$} & \multicolumn{2}{|c|}{$(\mathrm{R}=14-20)$} \\
\hline \multirow{2}{*}{$\begin{array}{c}\text { Exclusión }(\mathrm{R}= \\
0-8)\end{array}$} & 290 & 40,3 & 232 & 32,2 & 198 & 27,5 \\
\hline & \multicolumn{2}{|c|}{$(\mathrm{R}=0-2)$} & \multicolumn{2}{|c|}{$(\mathrm{R}=3-5)$} & \multicolumn{2}{|c|}{$(\mathrm{R}=6-8)$} \\
\hline
\end{tabular}

Nota. Los juicios se realizaron en escalas de 5 puntos $(0=$ Nada de acuerdo, $4=$ Muy de acuerdo $)$. Los valores entre paréntesis indican el rango de puntuación posible para cada variable. $\mathrm{N}=720 . \mathrm{f}=$ frecuencia. 


\subsection{DERECHOS BÁSICOS Y POLÍTICAS DE IGUALDAD}

En Derechos Básicos, los resultados del análisis de frecuencias, señalan que la mayoría de los individuos $(60,8 \%)$ manifiestan una actitud positiva hacia las políticas que garanticen los derechos fundamentales de educación, salud y movilidad de las personas inmigrantes. Alrededor de un tercio de los sujetos, muestra una actitud intermedia, pues puntúan entre 5 y 11. Es decir, no se manifiesta a favor o en contra de la aplicación de estos derechos.

En la variable Políticas de Igualdad, el rango de puntuación posible varía entre 0 y 24. Según los resultados presentados en la Tabla 2, destaca la actitud intermedia de los sujetos $(56,3 \%)$ sobre la actitud positiva (30\%). Así, más de la mitad de los individuos no muestran una postura definida, en relación con la implementación de iniciativas públicas que aseguren la igualdad de oportunidades para los inmigrantes ("Los inmigrantes deben tener los mismos derechos para acceder a una vivienda", "Los inmigrantes que residen en nuestro país, deben tener derecho a votar en las elecciones", "Estoy de acuerdo con una ley que permita cobrar el subsidio por desempleo a los inmigrantes"....).

\subsection{RASGOS POSITIVOS Y NEGATIVOS ASOCIADOS A LOS INMIGRANTES}

En Rasgos Positivos, el puntaje puede variar entre 0 y 12 . Alrededor del $25 \%$ de los sujetos puntúan entre 0 y 3 , por lo tanto, manifiestan percepciones o sentimientos positivos hacia los inmigrantes ("Los inmigrantes son gente honesta", "Los inmigrantes son gente educada" y "Los inmigrantes son gente trabajadora"). No obstante, en esta variable la mayor frecuencia de respuestas se situó en el rango 4-8 (70,5\%), lo que indica que más de dos tercios de los sujetos manifiestan una actitud intermedia, es decir, explícitamente no manifiestan sentimientos negativos hacia los inmigrantes.

En la variable Rasgos Negativos, el 55,8\% de los sujetos muestra una actitud positiva, el 43,3\% una actitud intermedia y sólo el 0,9\% una actitud negativa. Es decir, más de la mitad de los participantes no comparten la idea de que las personas inmigrantes se caracterizan por ser intolerantes, de poco fiar, no muy inteligentes o irresponsables. No obstante, más de un tercio de los sujetos muestra una actitud intermedia, es decir, no se manifiesta ni a favor ni en contra de las sentencias valoradas.

\subsection{ACEPTACIÓN Y CONVIVENCIA}

En la variable Actitud de Tolerancia, la mayor frecuencia se sitúa en el rango de puntuaciones que varía entre 9 y $12(44,5 \%)$ de un continúo que oscila entre 0 y 12 . Un porcentaje similar de individuos $(42,2 \%)$ puntuó en el rango que varía entre 4 y 8 . Así, de los resultados se desprende que alrededor de la mitad de los individuos bien muestra una actitud negativa o bien muestra una actitud intermedia respecto a tener un compañero de trabajo inmigrante o ser atendido por un médico inmigrante o tener como vecinos a un grupo de inmigrantes.

En Actitud de Aproximación, los resultados del análisis de frecuencias señalan que el $71,5 \%$ de los sujetos tiene una actitud intermedia, pues puntúa entre 3 y 5 y que otro porcentaje importante $(20,9 \%)$ muestra una actitud negativa que puntúa entre 6 y 8 . Es decir, la mayoría de los participantes bien no tienen una postura definida o bien evitan 
mostrar su disposición para establecer relaciones interpersonales íntimas, como casarse o mantener relaciones sexuales, con personas inmigrantes.

En la variable Actitud de Rechazo, el rango posible de puntuación puede oscilar entre 0 y 20. Según se aprecia en la Tabla 2, la gran mayoría de los sujetos $(81,7 \%)$, puntúan entre 0 y 6 , lo que indica que desestima la posibilidad de rechazar o evitar las relaciones esporádicas con los inmigrantes, como por ejemplo, ir a la piscina si hubiera inmigrantes, comprar a vendedores ambulantes inmigrantes, sentarse al lado de un inmigrante o sentir algún grado de afecto hacia éstas personas. Sólo un porcentaje muy inferior de sujetos $(0,5 \%)$ manifiesta una actitud negativa ya que sus respuestas se sitúan en el rango de puntuación que oscila entre 14 y 20.

En la variable Exclusión, las puntuaciones pueden variar entre 0 y 8 . La mayoría de los individuos puntúa entre 0 y $2(40,3 \%)$, lo cual indica que poco más de un tercio de los participantes percibe o está en conocimiento de que en Chile, la discriminación por motivos culturales es un problema real y que los grupos xenófobos existentes en el país son una amenaza para los inmigrantes. No obstante, según los resultados presentados en la Tabla 2, alrededor de otro tercio de sujetos $(27,5 \%)$ opina lo contrario.

En síntesis, los resultados de las actitudes hacia la inmigración señalan tendencias disímiles. Por un lado, la mayoría de los participantes muestra una tendencia positiva para compartir espacios públicos con los inmigrantes e incluso para establecer relaciones de amistad con estas personas. Además, reconocen a los inmigrantes como ciudadanos con derechos y desestiman los estereotipos negativos que se les puedan atribuir. También son capaces de reconocer que en Chile existe cierto grado de discriminación hacia los inmigrantes. No obstante, por otra parte, estos mismos sujetos no muestran una actitud claramente positiva o favorable para relacionarse íntimamente con los inmigrantes, ni son capaces de reconocer abiertamente algunas características positivas de estas personas, así como tampoco se muestran muy decididos a apoyar iniciativas de igualdad de oportunidades que favorezcan a los inmigrantes.

Para evaluar si existen diferencias significativas en las actitudes de los participantes en función de la experiencia de relación con personas inmigrantes (se han relacionado o no se han relacionado con ellos), en función del sexo y en función de la edad de los futuros profesores, se llevó a cabo análisis diferenciales empleando la prueba t de Student para muestras independientes.

Los resultados de la prueba de Levene que evalúa la igualdad de varianzas poblacionales (ver la Tabla 3), definiendo como variable independiente o de agrupamiento el sexo, la experiencia de relación con personas inmigrantes y la edad y como variables dependientes o agrupadas las actitudes hacia la inmigración (derechos básicos, políticas de igualdad, rasgos positivos, rasgos negativos, actitud de tolerancia, actitud de aproximación, actitud de rechazo y exclusión), señalan que, en función del sexo, si exceptuamos la variable exclusión, las demás no presentan diferencias significativas en las varianzas, lo cual permite asumir la homogeneidad poblacional. En función de la experiencia de relación, los resultados muestran que en la mitad de las variables es posible asumir la homogeneidad de varianzas (políticas de igualdad, rasgos negativos, actitud de tolerancia y exclusión) y en función de la edad, los resultados señalan que las variables no presentan diferencias significativas en las varianzas, lo cual permite asumir la homogeneidad de las mismas. 
Tabla 3. Prueba de Levene para la homogeneidad de varianzas. Sexo, experiencia de relación con inmigrantes y edad como variable dicotómica de agrupamiento y actitudes hacia la inmigración como variables agrupadas

\begin{tabular}{|c|c|c|c|c|c|}
\hline Variable & $\boldsymbol{F}$ & $p$ & Variable & $\boldsymbol{F}$ & $p$ \\
\hline Sexo & & & $\begin{array}{l}\text { Experiencia de } \\
\text { relación }\end{array}$ & & \\
\hline Derechos básicos & 1,50 & 0,221 & $\begin{array}{l}\text { Actitud de } \\
\text { tolerancia }\end{array}$ & 0,54 & 0,463 \\
\hline $\begin{array}{l}\text { Políticas de } \\
\text { igualdad }\end{array}$ & 0,28 & 0,599 & $\begin{array}{c}\text { Actitud de } \\
\text { aproximación }\end{array}$ & 6,38 & $0,012 *$ \\
\hline Rasgos positivos & 0,16 & 0,688 & Actitud de rechazo & 13,42 & $0,001 *$ \\
\hline Rasgos negativos & 0,13 & 0,720 & Exclusión & 0,07 & 0,789 \\
\hline $\begin{array}{l}\text { Actitud de } \\
\text { tolerancia }\end{array}$ & 0,19 & 0,663 & Edad & & \\
\hline $\begin{array}{c}\text { Actitud de } \\
\text { aproximación }\end{array}$ & 0,25 & 0,614 & Derechos básicos & 0,00 & 0,952 \\
\hline Actitud de rechazo & 1,78 & 0,183 & $\begin{array}{c}\text { Políticas de } \\
\text { igualdad }\end{array}$ & 1,42 & 0,233 \\
\hline Exclusión & 4,29 & $0,039 *$ & Rasgos positivos & 0,80 & 0,370 \\
\hline $\begin{array}{c}\text { Experiencia de } \\
\text { relación }\end{array}$ & & & Rasgos negativos & 0,67 & 0,415 \\
\hline Derechos básicos & 8,01 & $0,005^{*}$ & $\begin{array}{l}\text { Actitud de } \\
\text { tolerancia }\end{array}$ & 0,07 & 0,799 \\
\hline $\begin{array}{c}\text { Políticas de } \\
\text { igualdad } \\
\end{array}$ & 22,40 & $0,001 *$ & $\begin{array}{c}\text { Actitud de } \\
\text { aproximación }\end{array}$ & 1,15 & 0,284 \\
\hline Rasgos positivos & 1,87 & 0,172 & Actitud de rechazo & 0,47 & 0,492 \\
\hline Rasgos negativos & 0,81 & 0,369 & Exclusión & 0,01 & 0,911 \\
\hline
\end{tabular}

$* \mathrm{p}<0,05$.

En la Tabla 4 se presenta el análisis de contraste de medias en función de la experiencia de relación con personas inmigrantes. Los resultados señalan que existen diferencias significativas en las variables, Derechos básicos, Políticas de igualdad, Rasgos negativos, Rasgos positivos y Actitud de rechazo. De estos resultados se desprende que los participantes que sí se han relacionado con personas inmigrantes $(73,7 \%)$ en relación con aquellos participantes que no se han relacionado o no conocen personas inmigrantes $(26,3 \%)$, muestran una mejor actitud para apoyar políticas públicas que garanticen los derechos fundamentales, así como también una mejor disposición para apoyar iniciativas de igualdad de oportunidades hacia estas personas. Además, muestran una tendencia más positiva para establecer relaciones esporádicas con los inmigrantes, por lo tanto, también era de esperar que mostraran una percepción más positiva de los inmigrantes. 
Los análisis de contraste de medias en función de la edad de los sujetos señalaron que no se presentan diferencias estadísticamente significativas en las actitudes de los futuros maestros.

Por otra parte, los resultados del contraste de medias en función del sexo señalaron que existen diferencias significativas en la variable Actitud de aproximación ( $\mathrm{t}(718)=$ $3,52 ; \mathrm{p}<0,001)$. Esto indica que los hombres $(\mathrm{M}=4,22)$ en relación con las mujeres $(\mathrm{M}=4,61)$, muestran una mejor actitud para casarse o mantener relaciones sexuales con personas inmigrantes, es decir, los hombres estarían más o mejor dispuestos a establecer relaciones íntimas con estas personas.

Tabla 4. Contrastes de medias en función de la experiencia de relación con personas inmigrantes

\begin{tabular}{|c|c|c|c|c|c|c|c|}
\hline Variable & \multicolumn{4}{|c|}{ Descriptivo de grupo } & \multicolumn{3}{|c|}{ Pruebat } \\
\hline \multirow[t]{3}{*}{ Derechos básicos } & Relación & $\mathrm{n}$ & M & $\mathrm{DE}$ & $\mathrm{t}$ & $\mathrm{gl}$ & $\mathrm{p}$ \\
\hline & No & 261 & 4,66 & 3,83 & \multirow[t]{2}{*}{3,57} & \multirow[t]{2}{*}{473,75} & \multirow[t]{2}{*}{0,000 * } \\
\hline & Sí & 459 & 3,65 & 2,27 & & & \\
\hline \multirow[t]{2}{*}{ Políticas de igualdad } & No & 261 & 10,94 & 5,47 & \multirow[t]{2}{*}{2,06} & \multirow[t]{2}{*}{718} & \multirow[t]{2}{*}{$0,040 *$} \\
\hline & Sí & 459 & 10,09 & 5,24 & & & \\
\hline \multirow[t]{2}{*}{ Rasgos positivos } & No & 261 & 5,78 & 1,77 & \multirow[t]{2}{*}{7,18} & \multirow[t]{2}{*}{615,74} & \multirow[t]{2}{*}{$0,000 *$} \\
\hline & Sí & 459 & 4,73 & 2,09 & & & \\
\hline \multirow[t]{2}{*}{ Rasgos Negativos } & No & 261 & 6,73 & 3,38 & \multirow[t]{2}{*}{5,59} & \multirow[t]{2}{*}{718} & \multirow[t]{2}{*}{$0,000 *$} \\
\hline & $\overline{\text { Sí }}$ & 459 & 5,30 & 3,24 & & & \\
\hline \multirow[t]{2}{*}{ Actitud de tolerancia } & No & 261 & 7,72 & 3,50 & \multirow[t]{2}{*}{$-0,67$} & \multirow[t]{2}{*}{718} & \multirow[t]{2}{*}{0,501} \\
\hline & Sí & 459 & 7,91 & 3,68 & & & \\
\hline \multirow[t]{2}{*}{ Actitud de aproximación } & $\mathrm{No}$ & 261 & 4,51 & 1,26 & \multirow[t]{2}{*}{0,40} & \multirow[t]{2}{*}{599,78} & \multirow[t]{2}{*}{0,689} \\
\hline & Sí & 459 & 4,47 & 1,44 & & & \\
\hline \multirow[t]{2}{*}{ Actitud de rechazo } & No & 261 & 3,57 & 3,97 & \multirow[t]{2}{*}{3,64} & \multirow[t]{2}{*}{461,87} & \multirow[t]{2}{*}{$0,000 *$} \\
\hline & $\overline{\text { Sí }}$ & 459 & 2,51 & 3,29 & & & \\
\hline \multirow[t]{2}{*}{ Exclusión } & No & 261 & 3,78 & 2,77 & \multirow[t]{2}{*}{1,19} & \multirow[t]{2}{*}{718} & \multirow[t]{2}{*}{0,235} \\
\hline & $\overline{\text { Sí }}$ & 459 & 3,53 & 2,76 & & & \\
\hline
\end{tabular}

Cuanto más baja es la puntuación media (M), más positiva es la actitud.

$* \mathrm{p}<0,05$.

\section{DISCUSIÓN}

Los resultados señalan que los sujetos presentan distintos niveles de prejuicio cultural, en función de la cuestión que se valore. Al parecer, bajos niveles de prejuicio se asocian, principalmente, con comportamientos o acciones pasivas, es decir, no constituyen una relación directa y personal con las personas inmigrantes, como, por ejemplo, compartir espacios públicos, mostrarse a favor de que otros creen políticas que favorezcan a estos colectivos, aceptar que otros chilenos discriminan a los inmigrantes o que otros podrían 
atentar contra la integridad de los mismos. Por el contrario, altos niveles de prejuicio se vinculan con potenciales relaciones estrechas o de confianza entre los sujetos y las personas inmigrantes, a saber, compartir un espacio laboral, vivir en vecindad o recibir los servicios profesionales de un inmigrante.

Por otra parte, las actitudes intermedias se relacionan con la idea de relacionarse en forma activa con personas inmigrantes, con la manifestación de sentimientos positivos hacia estos y con la idea de brindarles igualdad de oportunidades. Creemos que esta actitud se relaciona, en alguna medida, con la representación moderna que ha adoptado el prejuicio, es decir, con esa forma enmascarada e indirecta de hostilidad hacia el exogrupo (Dovidio y Gaertner, 1986; Kinder y Sears, 1981; McConahay y Hough, 1976; Pettigrew y Meertens, 1995) que, en este caso en particular, se caracteriza, principalmente, por una tendencia a evitar las relaciones íntimas con los inmigrantes y por la falta de sentimientos positivos hacia estos. Esta actitud intermedia adopta una mayor relevancia considerando que los participantes son futuros maestros que, próximamente, no sólo deberán trasmitir y desarrollar nuevos aprendizajes, sino que también deberán traspasar a sus alumnos el acervo cultural y, con ello, las pautas sociales sobre las relaciones intergrupales y estimularlos a conocer y a respetar las diversas formas culturales que existen en Chile y en el mundo.

Es razonable pensar que esta actitud intermedia se sustenta y favorece, principalmente, en los principios normativos específicos que rigen a la sociedad chilena, es decir, que van en contra del prejuicio y de la discriminación explícita (Falomir et al., 2004; Muñoz et al. 2000; Sánchez-Mazas, Mugny, y Jovanovic, 1996) y que de alguna forma ejercen su influencia en las percepciones acerca de lo socialmente deseable. Debemos recordar que la deseabilidad social puede enmascarar actitudes latentes de mayor tendencia xenófoba (Morales, 2003) y que se ha observado que aquellos individuos con actitudes moderadas o ambivalentes presentan una mayor dependencia del contexto social que aquellos individuos con actitudes extremas (Hodson, Maio, y Esses, 2001; Mugny et al., 1991). Esto permite suponer que las normas sociales, ya sean favorables o desfavorables a la inmigración y a la interculturalidad, ejercerán una influencia significativa en aquellos individuos que manifiesten actitudes intermedias, como es el caso de los sujetos de este estudio.

De lo anterior se puede inferir que la actitud del profesor frente a una situación educativa intercultural, dependerá en cierta forma del conocimiento que tenga de las distintas culturas presentes en el aula, en el sentido de emplear una mayor o menor cantidad de suposiciones o ideas preconcebidas (estereotipos) para elaborar una representación cognitiva del alumno y derivar de éstas, expectativas sobre su rendimiento académico y actuar en consecuencia. Cabe recordar que las explicaciones más recientes de por qué los estudiantes de minorías étnicas tienen problemas en la escuela, otorgan un lugar importante a las bajas expectativas, a los prejuicios y a los estereotipos de los maestros (Navas, Holgado y Sánchez, 2009; Woolfolk, 2006) y que uno de los problemas causados por los estereotipos culturales dirigidos a los alumnos es la llamada "amenaza del estereotipo" que, en última instancia, interfiere las interacciones sociales y las tareas mentales de los estudiantes que lo padecen (Aronson, Fried, y Good, 2002; Steele, 1997).

En cuanto a la experiencia de relación con personas inmigrantes, se observaron diferencias estadísticamente significativas, es decir, el hecho de relacionarse o conocer personas inmigrantes introduce diferencias en las actitudes de los sujetos en el sentido que éstas se muestran más positivas. Estas diferencias concuerdan con la hipótesis que 
plantea que un mayor contacto con el exogrupo favorece el desarrollo de actitudes más positivas hacia sus miembros (Allport, 1954; Kalin y Berry, 1982; Pettigrew y Tropp, 2000).

Recogiendo las indicaciones de distintos autores, entre ellos, Cottrell y Neuberg (2005), Gaviria (2007), Molero (2007), Rodríguez, Betancor y Delgado (2009) y Smith (1993), creemos que en futuras investigaciones se debe intentar diferenciar los sentimientos que generan los distintos grupos culturales presentes en la sociedad chilena (peruanos, argentinos, colombianos, etcétera), ya que, al parecer, estos pueden dar lugar a diferentes conductas. Además, reconociendo la importancia de las normas sociales sobre el prejuicio y la discriminación y su influencia en las personas, creemos que es necesario determinar cuáles son las variables que están mediando para que en unos casos la norma permita criticar explícitamente a unos grupos, pero no a otros.

Por otra parte, si queremos entender y referirnos con propiedad a las relaciones entre los grupos, entonces la visión que tienen los componentes de los grupos discriminados es fundamental para estudiar el prejuicio y la discriminación en su conjunto.

Igualmente interesante sería valorar las respuestas considerando otras variables, analizar, por ejemplo, si la ideología política de los participantes, si su visión del mundo (global o local) o si la percepción subjetiva de bonanza o estabilidad económica del país introducen diferencias en sus actitudes hacia la inmigración, tal como se ha visto en otros contextos (Haubert y Fusell, 2006; Ward y Masgoret, 2008; Wilkes, Guppy y Farris, 2008).

Para finalizar, como implicación práctica derivada de los resultados, cabe plantearse la posibilidad de llevar a cabo un programa para el cambio de actitudes de los futuros maestros porque la presencia de actitudes intermedias en distintos aspectos relacionados con las actitudes hacia la inmigración sugiere la existencia del prejuicio moderno o sutil y porque, además, se observa una clara resistencia a establecer relaciones estrechas y de confianza con personas inmigrantes. Actitud que probablemente influirá en las relaciones profesor-alumno o profesor-padres, las mismas que tendrán que establecer los futuros maestros en situaciones educativas interculturales.

\section{REFERENCIAS BIBLIOGRÁFICAS}

Aboud, F. (1998). Children and prejudice. Londres: Blackwell Publishers.

Allport, G. (1954). The nature of prejudice. Reading: Adisson-Wesley.

Aronson, J., Fried, C., \& Good, C. (2002). Reducing the effecys of strereotype threat on African American college students: The role of theories of intelligence. Journal of Experimental Social Psychology, vol. 38, 113-125.

INSTITUTO NACIONAL DE ESTADísticas (2003). Características demográficas. En Instituto Nacional de Estadísticas, Censo 2002. Síntesis de resultados (pp. 9-19). Santiago, Chile. En línea; disponible en http//www.ine.cl/cd200sintesiscensal.pdf., el 30 de enero de 2009.

Presidencia de la República (2008). Política Nacional Migratoria. Santiago, Chile. En línea; disponible enhttp//www.interior.gov.cl/filesapp/Politica_Nacional_Migratoria.pdf, el 1 de febrero de 2009.

Cotrell, C. A., \& Neuberg, S. L. (2005). Different emotional reactions to different groups: A sociofunctional threat-based approach to "prejudice". Journal of Personality and Social Psychology, vol. $88,770-789$.

Díaz, A. M. (2002). Interacción y multiculturalidad en la escuela. En P. Pardo Y L. Méndez (Coords.), Psicología de la educación multicultural (pp. 279-309). Madrid: UNED. 
Díaz, M., Baraja, A. y Royo, P. (1996). Estudio sobre la integración escolar de alumnos extranjeros y el aprendizaje del castellano como segunda lengua. En M. Díaz (Coord.), Escuela y tolerancia (pp. 101-154). Madrid: Pirámide.

Dovidio, J. F., \& Gaertner, S. L. (1986). The aversive form of racism. En J. F. Dovidio \& S. L. GAERTNER (Eds.), Prejudice, discrimination, and racism (pp. 61-89). Orlando: Academic Press.

Falomir, J., Muñoz, D., Invernizzi, F., \& Mugny, G. (2004). Perceived in-group threat as a factor moderating the influence of in-group norms on discrimination against foreigners. European Journal of Social Psychology, vol. 34, 135-153.

Gallimore, R., \& Goldenberg, C. (2001). Analyzing cultural models and settings to connect minority achievement and school improvement research. Educational Psychologist, vol. 36, 45-56.

Gaviria, E. (2007). Los procesos psicosociales desde la perspectiva evolucionista. En J. F. Morales, M. Moya, E. Gaviria E. I. Cuadrado (Coords.), Psicología social (pp. 33-62). Madrid: McGraw Hill.

Glick, J., \& Hohmann-Marriott, B. (2007). Academic performance of young children in immigrant families. The significance of race, ethnicity, and national origins. International Migration Review, vol. 41, n. 2, 371-402.

Glick, J., \& White, M. (2003). The academic trajectories of immigrant youth. Analysis within and across cohorts. Demography, vol. 40, 749-784.

Haubert, J., \& Fusell, E. (2006). Explaining Pro-immigrant sentiment in the U. S.: Social Class, Cosmopolitanism, and Perceptions of Immigrants. International Migration Review, vol. 40, 489-507.

Hodson, G., Maio, G., \& Esses, V. (2001). The role of attitudinal ambivalence in susceptibility to consensus information. Basic and Applied Social Psychology, vol. 23, 197-205.

Holgado, P., Sánchez, A. y Navas, L. (2011). Análisis de la estructura de la Escala de Actitudes hacia la Inmigración en población chilena. Revista Iberoamericana de Diagnóstico y Evaluación Psicológica, vol. 29, n. 1, 97-113.

Jordan, J. (1994). La escuela multicultural. Barcelona: Paidós.

Kalin, R., \& Berry, J. (1982). The social ecology of ethnic attitudes in Canadian. Canadian Journal of Behavioral Science, vol. 25, 28-44.

Kinder, D., \& Sears, D. (1981). Symbolic racism versus racial threats to the good life. Journal of Personality and Social Psychology, vol. 40, 414-431.

León, B., Mira, A. y Gómez, T. (2007). Evaluación de las opiniones sobre la inmigración y la multiculturalidad en la escuela de alumnos de magisterio. Revista Electrónica de Investigación Psicoeducativa, vol. 5, n. 2, 259-282.

Mcconahay, J., \& Hough, J. (1976). Symbolic racism. Journal of Social Issues, vol. 32, 23-45.

Molero, F. (2007). El estudio del prejuicio en la psicología social: definición y causas. En J. F. Morales, M. Moya, E. Gaviria E I. Cuadrado (Coords.), Psicología social (pp. 591-617). Madrid: McGraw-Hill.

Mugny, G., Sánchez-Mazas, M., Roux, P., \& Pérez, J. (1991). Independence and interdependence and interdependence of group judgements: Xenophobia and monority influence. European Journal Of Social Psychology, vol. 21, 213-223.

Muñoz, D., Falomir, J., Invernizzi, F., \& Leuenberger, S. (2000). Normative discrepancies and social discrimination change in an experimental group setting. International Review of Social Psychology, vol. 13, 7-40.

Navas, L., Holgado, P. y Sánchez, A. (2009). Predicción de los estereotipos académicos ante los estudiantes inmigrantes. Horizontes Educacionales, vol. 14, n. 2, 37-47.

Pettigrew, T., \& Meertens, R. W. (1995). Subtle and blantant prejudice in Western Europe. European Journal of Social Psychology, vol. 25, 57-75.

Pettigrew, T., \& Tropp, L. (2000). Does Intergroup Contac Reduce Prejudice: Recent Meta-Analytic Findings. En S. Oskam (Ed.), Reducing Prejudice and Discrimination (pp. 93-114). Mahwah, NJ: Lawrence Erlbaum. 
Porter, A., \& Macleod, D. (1996). Educational progress of children of immigrant: The roles of class, ethnicity, and school context. Sociology of Education, vol. 69, 255-275.

Quesille, A. (2007). Inmigrantes, Refugiados y Derechos Humanos. En N. Espejo (Coord.), Informe anual sobre derechos humanos en Chile. Hechos 2006 (pp. 317-362). Santiago de Chile: Universidad Diego Portales.

Rodríguez, A., Betancor, V. y Delgado, N. (2009). La norma social sobre la expresión de prejuicio explícito hacia diferentes grupos sociales. Revista de Psicología Social, vol. 24, n. 1, 17-27.

Sánchez-Mazas, M., Mugny, G., \& Jovanovic, J. (1996). Conflict normatif et changement des attitudes intergroups. International Review of Social Psychology, vol. 8, 25-43.

Smith, E. R. (1993). Social identity emotions: Toward new conceptualizations of prejudice. En D. M. Mackie \& D. L. Hamilton (Eds.), Affect, cognition and stereotyping. Interactive processes in group perception (pp. 297-315). Nueva York: Academic Press.

Steele, C. (1997). A threat in the air: How stereotypes shape intellectual identity and performance. American Psychologist, vol. 52, 613-629.

Ward, C., \& Masgoret, A. (2008). Attitudes toward Immigrants, Immigration, and Multiculturalism in New Zealand: A Social Psychological Analysis. International Migration Review, vol. 42, n.1, 227-248.

Wilkes, R., Guppy, N., \& Farris, L. (2008). "No Thanks, We're Full”: Individual Characteristic, National Context, and Changing Attitudes Toward Immigration. International Migration Review, vol. 42, n.2, 302-329.

Woolfolk, A. (2006). Psicología educativa. Ciudad de México: Pearson Education. 
